\title{
Hydro-Gravitational Dynamics of Planets and Dark Energy
}

\author{
C. H. Gibson ${ }^{1,2}$ and R. E. Schild ${ }^{3,4}$ \\ ${ }^{1}$ University of California San Diego, La Jolla, CA 92093-0411, USA \\ ${ }^{2}$ Email: cgibson@ucsd.edu, http://sdcc3.ucsd.edu/ ir118 \\ ${ }^{3}$ Center for Astrophysics, 60 Garden Street, Cambridge, MA 02138, USA \\ ${ }^{4}$ Email: rschild@cfa.harvard.edu
}

(Received December 29, 2007; accepted August 12, 2008)

\begin{abstract}
Self gravitational fluid mechanical methods termed hydro-gravitational-dynamics (HGD) predict plasma fragmentation $0.03 \mathrm{Myr}$ after the turbulent big bang to form protosuperclustervoids, turbulent protosuperclusters, and protogalaxies at the $0.3 \mathrm{Myr}$ transition from plasma to gas. Linear protogalaxyclusters fragment at $0.003 \mathrm{Mpc}$ viscous-inertial scales along turbulent vortex lines or in spirals, as observed. The plasma protogalaxies fragment on transition into white-hot planet-mass gas clouds (PFPs) in million-solar-mass clumps (PGCs) that become globular-star-clusters (GCs) from tidal forces or dark matter (PGCs) by freezing and diffusion into $0.3 \mathrm{Mpc}$ halos with $97 \%$ of the galaxy mass. The weakly collisional non-baryonic dark matter diffuses to $>\mathrm{Mpc}$ scales and fragments to form galaxy cluster halos. Stars and larger planets form by binary mergers of the trillion PFPs per PGC, mostly on $0.03 \mathrm{Mpc}$ galaxy accretion disks. Stars deaths depend on rates of planet accretion and internal star mixing. Moderate accretion rates pro-duce white dwarfs that evaporate surrounding gas planets by spin-radiation to form planetary nebulae before Supernova Ia events, dimming some events to give systematic distance errors, the dark energy hypothesis, and overestimates of the universe age.
\end{abstract}

\section{INTRODUCTION}

Dimness of supernovae Ia (SNe Ia) events for redshift values $0.01<\mathrm{z}<2$ have been interpreted as an accelerating expansion rate for the universe (Riess et al. 1998, Perlmuter et al. 1999, and Chernin et al. 2007). The acceleration is attributed to mysterious antigravity effects of "dark energy" and a cosmological constant $\Lambda$. Dimming is observed at all frequencies by about $30 \%$, with large scatter attributed to uncertainty in the SNe Ia models. Hubble Space Telescope Advanced Camera for Surveys (HST/ACS) images have such high signal to noise ratios that both the scatter and the dimming are statistically significant over the full range of $z$ values. Bright $\mathrm{SNe}$ Ia observed for $\mathrm{z} \geq 0.46$ exclude "uniform grey dust" systematic errors, supporting flatuniverse deceleration until the recent "cosmic jerk" to acceleration for $\mathrm{z} \leq 0.46$. The "dark energy" interpretation is a consequence of the commonly accepted $\Lambda$-cold-darkmatter $(\Lambda \mathrm{CDM})$ cosmological theory. However, $\Lambda \mathrm{CDM}$ theory is fluid mechanically untenable. The theory assumes irrotational, collisionless and frictionless flows, neglecting effects of viscosity, turbulence, diffusion, fossil turbulence and fossil turbulence waves.

Turbulence is defined as an eddy-like state of fluid motion where the inertial-vortex forces of the eddies are larger than any other forces that tend to damp the eddies out. Turbulence by this definition always cascades from small scales to large, starting at the viscous-inertial-vortex Kolmogorov scale at a universal critical Reynolds number. The mechanism of this «inverse» cascade is merging of adjacent vortices with the same spin due to induced inertial vortex forces that force eddy mergers at all scales of the turbulence. Such eddy mergers account for the growth of turbulent boundary layers, jets and wakes. A false myth of turbulence theory is that turbulence cascades from large scales to small. It never does, and could not be universally similar if it did. Irrotational flows cannot be turbulent by definition. In self-gravitational fluids such as stars and planets, turbulence is fossilized in the radial direction by buoyancy forces. Radial transport is dominated by fossil turbulence waves and secondary (zombie) turbulence and zombie turbulence waves in a beamed, radial, hydrodynamic-maser action.

Hydro-gravitational-dynamics (HGD) is the application of modern fluid mechanics to cosmology (Gibson 2008, 2006, 2005, 2004, 2000, 1996, Gibson and Schild 2007a, 2007b, 2002, and Schild and Gibson 2008). Primordial planets formed at $z=1100, \quad t=10^{13} \mathrm{~s}$ in protoglobularstarcluster clumps as predicted by HGD are the source of all stars and the dark matter of galaxies (Gibson 2008, 2006, 2005, 2004, 2000, 1996, Gibson and Schild 2007a, 2007b, 2002, Schild and Gibson 2008, and Schild 1996). Because dying stars may be dimmed by gas planets evaporated near the star, planets provide an alternative to dark energy. New physical laws are not required by HGD but $\Lambda$ CDM must be discarded. The choice is between planets and dark energy.

In the following Section 2 we discuss the theories of 
gravitational structure formation, followed by a review of the observational data in Section 3, a Discussion of Results in Section 4 and finally some Conclusions in Section 5.

\section{THEORY}

The Jeans 1902 linear theory of acoustic gravitational instability predicts only one criterion for structure formation. Fluctuations of density are assumed unstable at length scales larger than the Jeans length $L_{J}=V_{S} /(\rho G)^{1 / 2}$ but stable for smaller scales, where $V_{S}$ is the speed of sound, $\rho$ is density, $G$ is Newton's gravitational constant, and $(\rho G)^{-1 / 2}$ is the gravitational free fall time. Because the speed of sound in the plasma epoch after the big bang is nearly the speed of light, the Jeans scale for the plasma is always larger than the scale of causal connection $c t$, where $c$ is the speed of light and $t$ is the time since the big bang, so no gravitational structures can form in the plasma. Jeans' 1902 fluid mechanical model is the basis of $\Lambda \mathrm{CDM}$, where an unknown form of collisionless non-baryonic dark matter (NBDM) is assumed to condense because it is somehow created cold so its sound speed and Jeans length can be assumed smaller than ct. The NBDM clumps cluster hierarchically and magically stick together to form potential wells in $\Lambda$ CDMHC models (Riess et al. 1998 and Perlmuter et al. 1999) with dark energy. CDM clumps have been sought but not observed, as predicted by HGD.

It is not true that the primordial plasma is collisionless and it is not true that gravitational instability is linear. Gravitational instability is intrinsically nonlinear and absolute in the absence of viscosity just like the inertialvortex-force $\overrightarrow{\mathrm{v}} \times \stackrel{1}{\omega}$ turbulence instability is nonlinear and absolute in the absence of viscosity. All fluid density fluctuations are unstable to gravity, and in the plasma epoch $10^{11} \leq t \leq 10^{13} \mathrm{~s}$ structures will form by gravitational forces at all scales less than $c t$ unless prevented by diffusion, viscous forces or turbulence forces, as shown in Fig. 1 (Schild and Gibson 2008).

The kinematic viscosity of the plasma is determined by photon collisions with free electrons that drag their protons and alpha particles with them. The photon viscosity of the plasma epoch $v_{p} \approx 4 \times 10^{26} \mathrm{~m}^{2} \mathrm{~s}^{-1}$ (Gibson 2000) is so large that the potential wells of CDM halos have Reynold numbers less than one and could not fill with plasma in the available time, and any accoustic oscillations from the filling would be prevented by photon-viscosity. Thus the dominant accoustic peak of the CMB temperature anisotropy spectrum of Fig. 1a is explained by HGD theory from the sonic-speed expansion of protosupercluster voids triggered by plasma density minima as shown in Fig. $1 \mathrm{~b}$, but not by CDM theory if viscosity is included in the numerical simulations. The mean free path for photonelectron collisions is less than the horizon scale by two orders of magnitude at the time of first structure from the Thomson scattering cross section and the known electron density, as required by the continuum hypothesis of fluid mechanics. When the Schwarz viscous-gravitational scale $L_{S V}$ becomes less than the horizon scale $L_{H}=c t$ then gravitational structure formation begins.

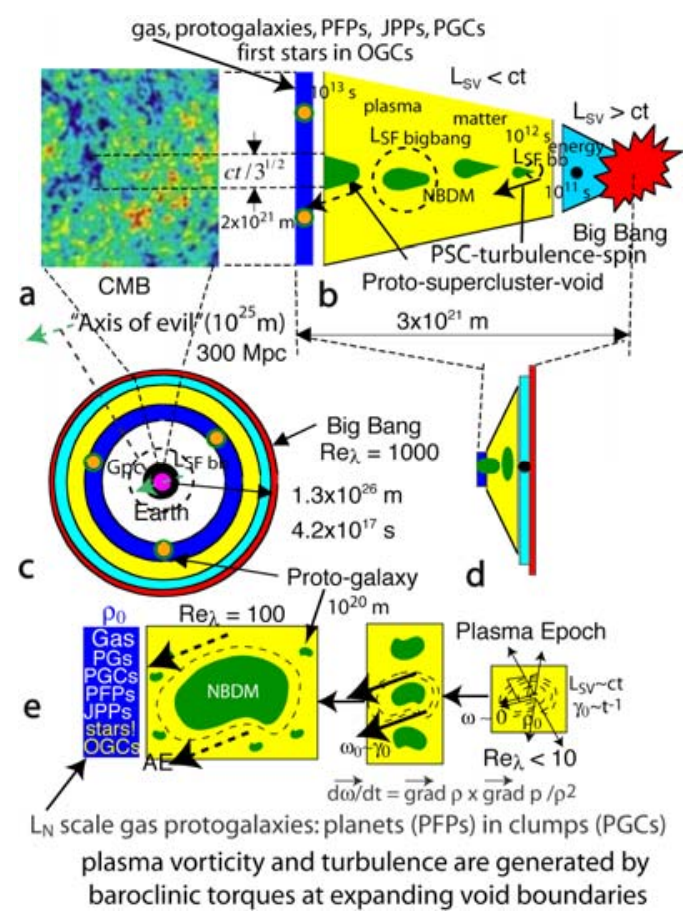

Fig. 1. Formation of gravitational structures according to hydro-gravitational-dynamics (HGD). The entire baryonic plasma universe fragments at the gas Schwarz viscous scale to form planets in Jeans mass clumps in Nomura geometry (Gibson 2008) protogalaxies at transition. Plasma fossil big bang turbulence density gradients produce "axis of evil" quasar, galaxy and galaxy cluster spin alignments to Gpc scales (Schild and Gibson 2008 and Hutsemekers et al. 2005) by baroclinic torques at the expanding void boundaries.

The first structures are fragmentations because the rapid expansion of the universe inhibits condensation at density maxima but unhances void formation at density minima. It is a myth of astrophysics that pressure-support or thermal-support will prevent structure formation at scales smaller than the Jeans scale $L_{J}=V_{s} /(\rho G)^{1 / 2}$. Pressure support occurs in hydrostatics, not hydrodynamics. At the plasma-gas transition the kinematic viscosity decreases by a factor of $\approx 10^{13}$, permitting fragmentation at gasplanetary rather than plasma-galactic scales $L_{S V}=(\gamma \nu / \rho G)^{1 / 2}$. Viscous forces are no longer able to prevent void growth at density minima so the protogalaxies fragment to form primordial fog particles. From the Bernoulli equation the pressure decreases during the fragmentation process as the specific enthalpy $p / \rho$ decreases to compensate for the increasing kinetic energy per unit mass $\mathrm{v}^{2} / 2$ along streamlines toward density maxima or away from density minima. Consider what happens when a cannonball or a vacuum beachball suddenly appears in a uniform motionless gas (Gibson 2000). In the first case gravity accelerates everything toward the center. In the second case gravity accelerates everything away. Nothing much happens until the gravitational free fall time, at which the density exponentiates to infinity or zero.

In either case the pressure gradient is in the direction of 
motion, opposite to that required for the fictitious pressure support mechanism. For gravitational condensations after a gravitational free-fall time $\tau_{F F}=(\rho G)^{-1 / 2}$ the density increases exponentially to form a planet or star with internal stresses to bring the condensation to a halt and allow hydrostatic equilibrium with pressure forces in balance with gravitational forces (Gibson 2000). For gravitational void formation $\tau_{F F}=(\rho G)^{-1 / 2}$ is the time required for the rarefaction wave of the growing void to reach its limiting speed at Mach 1 from the Rankine-Hugoniot relations, since rarefaction shocks are impossible from the second law of thermodynamics. This permits the prediction by HGD of the dominant size of $\mathrm{CMB}$ temperature anisotropies (Fig. 1a) to be $c t / 3^{1 / 2}$ or $1.7 \times 10^{21} \mathrm{~m}$ at time $t=10^{13} \mathrm{~s}$, as observed.

The density and rate-of-strain of the plasma at transition to gas at 300,000 years $\left(10^{13} \mathrm{~s}\right)$ are preserved as fossils of the time of first structure at 30,000 years $\left(10^{12} \mathrm{~s}\right)$, as shown in Fig. 1e. The plasma turbulence is weak at transition, so the Schwarz viscous scale $L_{S V}=(\gamma v / \rho G)^{1 / 2}$ and Schwarz turbulence scale $L_{S T}=\varepsilon^{1 / 2} /(\rho G)^{3 / 4}$ are nearly equal, where $\gamma$ is the rate-of-strain, $v$ is the kinematic viscosity, $\varepsilon$ is the viscous dissipation rate and $\rho$ is the density. Because the temperature, density, rate-of-strain, composition and thus kinematic viscosity of the primordial gas are all well known it is easy to compute the fragmentation masses to be that of protogalaxies composed almost entirely of $10^{24}-10^{25} \mathrm{~kg}$ planets in million-solarmass $10^{36} \mathrm{~kg}$ (PGC) clumps (Gibson 1996). The NBDM diffuses to diffusive Schwarz scales $L_{S D}=\left(D^{2} / \rho G\right)^{1 / 4}$ much larger than $L_{N}$ scale protogalaxies, where $D$ is the NBDM diffusivity and $D ? v$. The rogue-planet prediction of HGD was promptly and independently predicted by the Schild 1996 interpretation of his quasar microlensing observations (Schild 1996).

The HGD prediction of $\operatorname{Re}_{\lambda}: 100$ weak turbulence in the plasma epoch is supported by statistical studies of cosmic microwave background (CMB) temperature anisotropy fine structure compared to atmospheric, laboratory and numerical simulation turbulence values (Breshadskii 2006, Breshadskii and Sreenivasam 2002, 2003). Plasma turbulence imposes a preferred direction to the massive plasma objects formed by gravitational instability in the plasma at length scales that reflect fossil temperature turbulence of the big bang (Gibson 2004). Baroclinic torques produce vorticity at rate $\partial \stackrel{r}{\omega} / \partial t=\nabla \rho \times \nabla p / \rho^{2}$ at the boundary of protosupercluster voids as shown in Fig. 1e (Schild and Gibson 2008). Because $\nabla \rho$ is roughly constant over

$L_{S F \text { bigbang }}$ fossil turbulence scales of the big bang turbulence at strong force freeze-out stretched by inflation, HGD explains observations of quasar polarization matching the direction of the Axis of Evil to Gpc scales approaching the present horizon scale $L_{H}=c t$ (Hutsemekers et al.
2005). This direction on the cosmic sphere is right ascension RA $=202^{\circ}$, declination $\delta=25^{\circ}$, which matches the 2-4-8-16 directions of $\mathrm{CMB}$ spherical harmonics (Land and Magueijo 2005) and galaxy spins to supercluster $30 \mathrm{Mpc}$ scales (Gahm et al. 2007a), and is quite unexpected and inexplicable from $\Lambda \mathrm{CDM}$ theory. All temperature fluctuations of big bang turbulence are fossilized by inflation, which stretches space and the fluctuations beyond the scale of causal connection $c t$ at speeds $\approx 10^{25} C$ (Gibson 2004).

\section{OBSERVATIONS}

Figure 2 (top) shows the Tadpole (VV29, UGC 10214) galaxy merger system imaged by the HST/ACS camera, compared to a Keck Telescope spectroscopic study (bottom) by Tran et al. 2003 (Tran et al. 2003). The galaxy dark matter clearly consists of PGCs since the spectroscopy proves the YGCs were formed in place in the galaxy halo and not ejected as a collisionless tidal tail (Toomre et al. 1972). Quasar microlensing (Schild 1996) suggests the dark matter PGCs must be composed of frozen planets in metastable equilibrium, as predicted by HGD (Gibson 1996).

Figure 2 (bottom) shows a linear trail of YGCs pointing precisely to the frictional spiral merger of the small blue galaxy VV29c that is embedded in the accretion disk of VV29a, Fig. 2 (top). With tidal agitation from the merger, the planets undergo an accretional cascade to larger and larger planets, and finally form stars within the PGCs that are stretched away by tidal forces to become field stars in the observed star-wakes and dust-wakes.

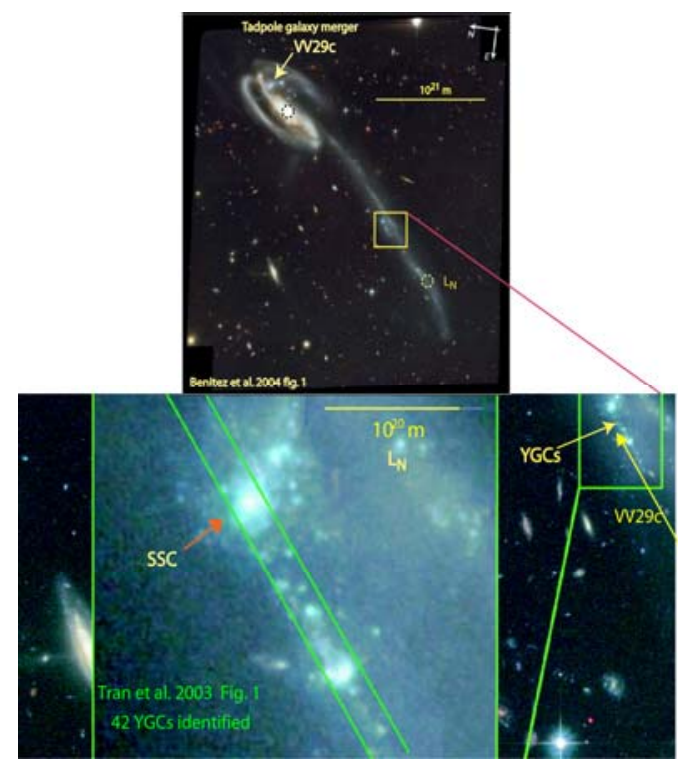

Fig. 2. Tadpole galaxy merger system illustrating the size of the baryonic dark matter system surrounding the central galaxy VV29a and the frictional spiral merger of galaxy VV29c leaving a trail of young-globular-star-clusters (YGCs)

All galaxies originate as protogalaxies at $0.03 \mathrm{Mpc}$ scales $L_{N}$ reflecting viscous-gravitational fragmentation of weakly turbulent plasma just before its transition to gas. 
A core of $13.7 \mathrm{Gyr}$ old stars at scale $L_{N}$ persists in most if not all galaxies bound by PGC-viscosity of its remaining PGC dark matter. Most of the PGC mass diffuses out of the protogalaxy core to form the galaxy dark matter halo, observed to extend to a diameter of $0.3 \mathrm{Mpc}$ in Tadpole, Fig. 1 (top). A more detailed discussion is found elsewhere (Gibson and Schild 2002).

Figure 3 shows a planetary nebula in the Large Magellanic Cloud (LMC) claimed from a recent brightness episode to be on the verge of a SNe Ia event (Pena et al. 2004), where the central white dwarf and companion (or possibly just a white dwarf and a JPP accretion disk) have ejected several solar masses of matter in the bright clumps observed. The HGD interpretation is very different (Gibson and Scild 2007a). From HGD the bright clumps are formed in place from clumped baryonic-dark-matter frozen planets termed JPPs (Jovian PFP Planets of all sizes form by gassy binary accretional mergers of PFPs and their growing daughters), where some of the multi-Jupiter-mass clumps (globulettes (Gahm et al. 2007b)) are accreted by the star, and none are ejected. As the JPPs are accreted the star shrinks, its mass and density increase, and its spin rate increases producing a powerful plasma beam that evaporates the frozen gas planets it encounters. Moderate JPP accretion rates may fail to mix away the carbon core giving a supernova Ia at the Chandresekhar critical mass 1.44 solar. Stronger mixing and accretion may permit an iron core and a supernova II event.

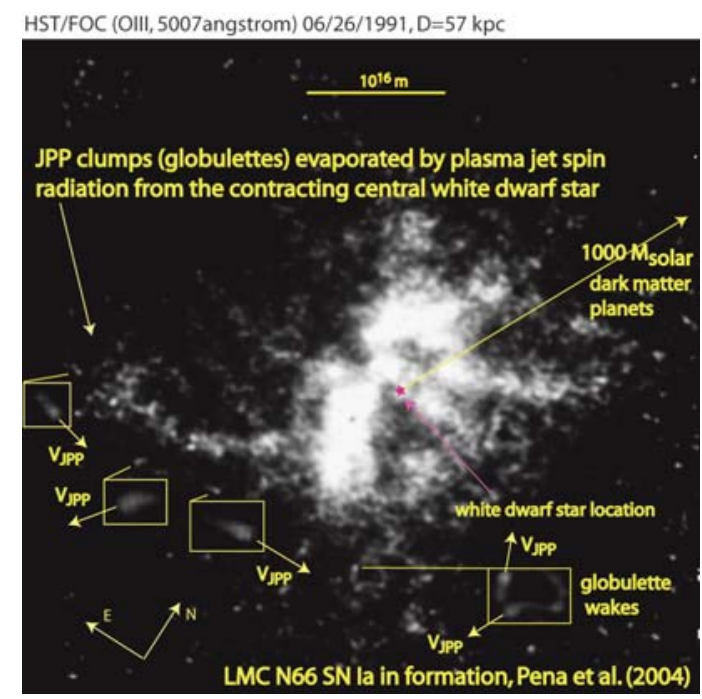

Fig. 3. Planetary nebula LMC N66 suggested by Pena et al. 2004 as a Supernova Ia in formation (Pena et al. 2004). From HGD the HST/FOC image reveals globulette clumps (Gahm et al. 2007b) of dark matter planets evaporated by the plasma jet of the rapidly spinning, contracting, white dwarf star at the center, burdened by the rain of accreting, evaporating, planets.

Even stronger accretion rates lead to superstars or black holes. Within the PNe size there should be more than a thousand solar masses of dark matter planets, as shown by the arrow toward upper right.

Figure 4 shows the Helix planetary nebula. Helix is much closer $(209 \mathrm{pc})$ and presumably less strongly agitated by tidal forces from other objects than the LMC N66 PNe of Fig. 3.

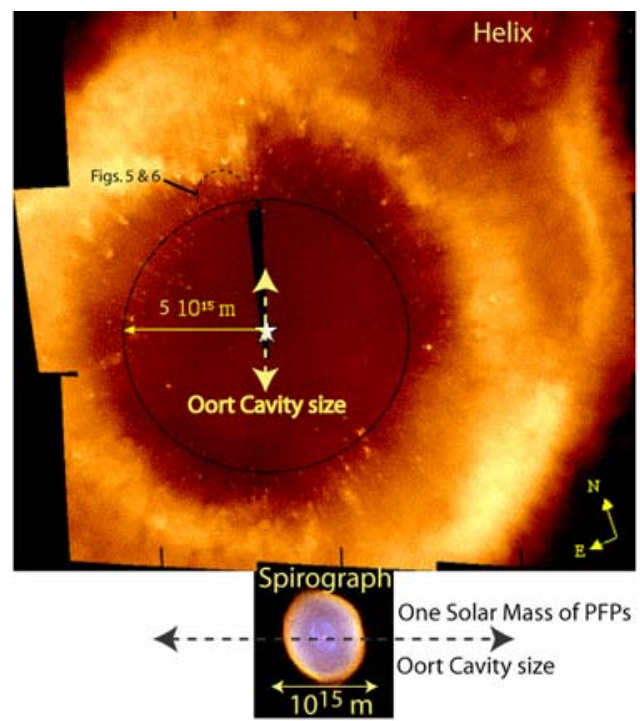

Fig. 4. Helix planetary nebula (top) showing numerous dark matter planets evaporated by the central white dwarf at the outer boundary of the Oort cavity left by the accretion of PFPs to form the star within the PGC. Spirograph PNe (bottom) is young and still growing within its Oort cavity, where apparently no dark matter planets remain unevaporated. Detailed images of Helix dark matter planets are shown in Fig. 5 and Fig. 6 at the indicated location north of the central star.

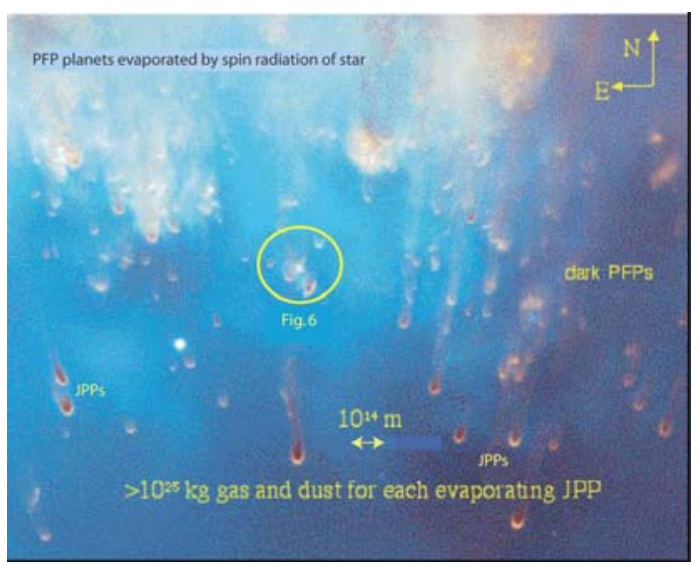

Fig. 5. Closeup image of the region north of the central star shown by the dashed circle in Fig. 4. The central white dwarf spins rapidly because it has shrunk to a density of order $10^{10} \mathrm{~kg} \mathrm{~m}^{-3}$ from the mass of accreted planets. A bipolar plasma beam irradiates and evaporates dark matter planets at the edge of the Oort cavity, creating the planetary nebula.

Thousands of evaporating gas planets can be seen in Fig. 4 (and Fig. 5 and Fig. 6 close-up) HST images. The planets have spacing consistent with the fossil density from the time of first structure at 30,000 years after the big bang; that is, $\rho_{0}: 10^{-17} \mathrm{~kg} \mathrm{~m}^{-3}$. 


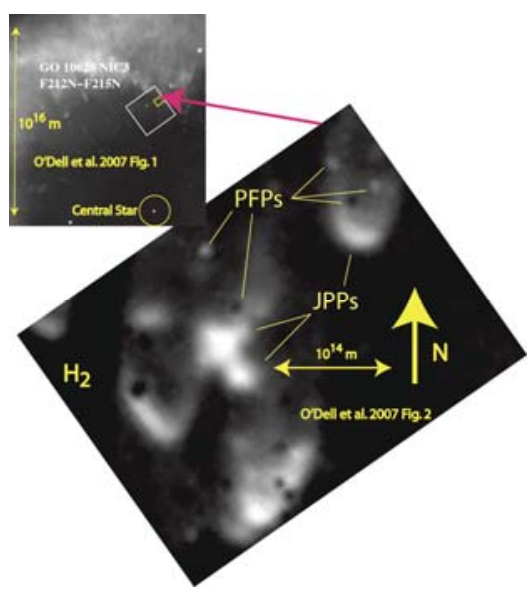

Fig. 6. Detail of location north of central white dwarf star in Helix PNe (O'Dell et al. 2007), showing evaporating PFP planets as well as large JPP planets and their atmospheres that can dim a SNe Ia event if it is along the line of sight, Fig. 8 .

The mass of white-dwarf-precursor stars are vastly overestimated by ignorance of the existence of dark matter planets. Without PFPs, standard PNe models assume the mass of the nebula originates in the original star and is somehow ejected as clumps and superwinds. Figure 6 shows close-up images of the JPP and partially evaporated PFP planets, illuminated by the spin-radiation of the central star.

Figure 7 shows estimates of the final mass of stars $M_{\text {Final }} \leq 1.3 M_{\text {sun }} \leq M_{\text {Pulsar }} \leq M_{\text {SNeIa }}$ compared to the initial mass $M_{P N E}$ where the PNe mass is estimated from the nebulae brightness assuming all of the nebula mass originates in the star and none from dark matter planets.

The brightest SNe Ia events agree with the no-dark-energy curve of Fig. 8 (top) and can be interpreted as lines of sight that do not intersect dense dark matter planet atmospheres. A similar interpretation is given to the Sandage et al. 2006 $\mathrm{SNe}$ Ia global Cepheid Hubble-Constant $H_{0}=62.3$ $\mathrm{km} \mathrm{s}^{-1} \mathrm{Mpc}^{-1}$ estimates that disagree with the WMAP $H_{0}=71 \mathrm{~km} \mathrm{~s}^{-1} \mathrm{Mpc}^{-1}$ value and estimate of the age of the universe $\mathrm{T}$ to be $15.9 \mathrm{Gyr}$ rather than the $\mathrm{CMB}$ value of 13.7 Gyr. Taking the least dim SNe Ia values measured to be correct removes the systematic error of dark matter planet atmosphere dimming (non-linear grey dust), so discrepancies in $\mathrm{T}$ and $H_{0}$ are removed.

We see from Fig. 7 that final star masses are much smaller than initial masses by standard models of PNe and $\mathrm{SNe}$. Pulsar masses $M_{\text {Pulsar }}=1.4 M_{\text {sun }}$ are less than the Chandrasekhar white dwarf limit $M_{\text {Chandrasekhar }}=1.44 M_{\text {sun }}$.

Figure 8 shows that an alternative to dark energy is dark matter planets. Figure 8 (top) is dimness of SNe Ia events vesus redshift $\mathrm{z}$ for uniform-grey-dust, dark-energy (nonlinear-grey-dust) and no-dark-energy models. The uniform-grey-dust model fails at large z. Dark matter planets provide a nonlinear-grey-dust effect if $\mathrm{SNe}$ Ia events take place in PNe surroundings such as the Helix, Fig. 8 (bottom) where spin-radiation of a white dwarf creates JPP atmospheres that may dim or not dim depending on the line of sight.

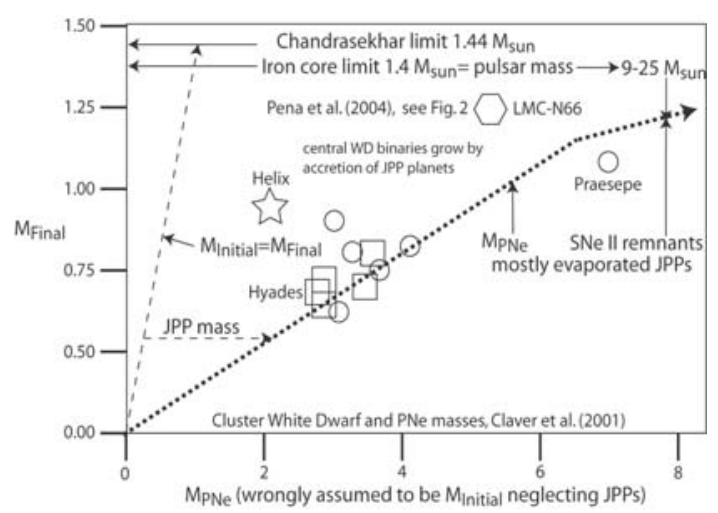

Fig. 7. Star masses compared to assumed $\mathrm{PNe}$ and $\mathrm{SNe}$ initial star masses neglecting dark matter planet effects such as JPP atmosphere brightness and the mass of evaporated dust and gas (Gibson and Schild 2007a).

\section{Discussion}

An accumulation of evidence in a variety of frequency bands from a variety of very high resolution and highly sensitive modern telescopes leaves little doubt that the dark matter of galaxies is primordial planets in protoglobular-star-cluster clumps, as predicted from HGD by Gibson 1996 and inferred from quasar microlensing by Schild 1996. All stars form from these planets so all star models and planetary nebulae models must be revised to take the effects of planets and their brightness and dimness effects into account. Turbulence produces postturbulence (fossil turbulence) with structure in patterns that preserve evidence of previous events such as big bang turbulence and plasma epoch turbulence. Post-turbulence perturbations (Gibson 2004) guide the evolution of all subsequent gravitational structures. Numerous fatal flaws in the standard LCDMHC cosmology have appeared that can be traced to inappropriate and outdated fluid mechanical assumptions (Jeans 1902) that can be corrected by HGD (Gibson 2008, 2006, 2005, 2004, 2000, 1996, Gibson and Schild 2007a, 2007b, 2002, Schild and Gibson 2008, and Schild 1996).

\section{Conclusions}

Dark matter planet dimming errors account for the SNe Ia overestimate $(\mathrm{T}=15.9 \mathrm{Gyr})$ of the age of the universe (Sandage et al. 2006) and dark energy dimming of SNe Ia events, as described by Fig. 8 .

Dark energy is an unecessary and incorrect hypothesis from HGD. Thus we need not modify any physical laws nor predict the end of cosmology because evidence of cosmological beginnings are being swept out of sight by an accelerating vacuum-antigravity-powered expansion of the universe (Krauss and Scerrer 2008, 2007). 


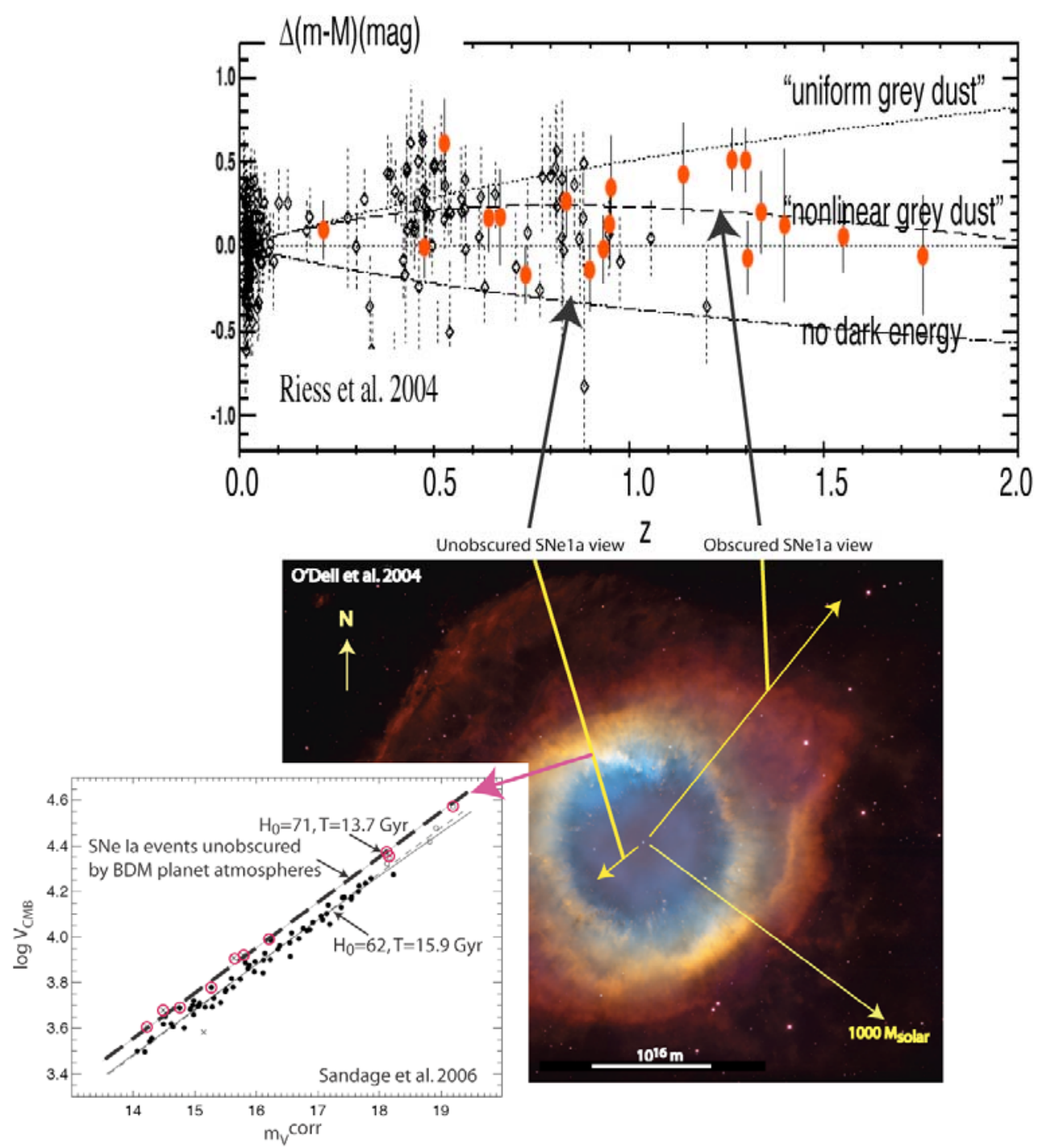

Fig. 8. Helix planetary nebula showing the effect of JPP planetary atmospheres along the line of sight to SNe Ia events is to produce a systematic dimming error that can masquerade both as dark energy (top) or as increased Hubble constants and ages of the universe (left insert). Open circles (red) are bright SNe Ia events of the Sandage 2006 data set taken to be unobscured by evaporated planetary atmospheres, and supporting the CMB universe age T = 13.7 Gyr.

From HGD and the second law of thermodynamics, the frictional non-adiabatic big bang turbulence beginning of the universe (Gibson 2005) implies the universe is closed, not open.

Because the big bang was extremely hot, the big bang turbulence produced little entropy so the departures from a flat universe should be small, as observed. Fossil strongforce freeze-out scale density turbulence explains the Gpc scales of quasar polarization vectors in alignment with the CMB axis of evil (Hutsemekers et al. 2005, Gahm et al. 2007a, Schild and Gibson 2008), as shown by the dashed circles in Fig. 1. Negative big bang turbulence stresses and negative gluon viscous stresses are candidates to drive the exponential inflation of space during and at the end of the big bang. An inflationary event is indicated by observations showing similar galaxy patterns exist in regions outside each others causal connection scales.
Star formation models and planetary nebulae formation models must be corrected to account for the effects of dark matter planets (Gibson and Schild 2007a). Initial star masses have been vastly overestimated from the brightness of evaporating planets formed around dying central stars by spin radiation, as shown by Fig. 7. Red giant and asymptotic giant branch (AGB) excursions of brightness on the Hertzsprung-Russell color-magnitude diagram for star evolution must be reexamined to account for the fact that all stars are formed and grow by the accretion of planets that have first order effects on both the color and magnitude of stars as they evolve. Planetary nebulae are apparently not dust clouds ejected by AGB superwind events as usually assumed but are partially evaporated and brightly illuminated dark matter planets, as shown by Figs. 3-6, and Fig. 8 . 
C.H. Gibson and R.E. Schild/ JAFM, Vol. 2, No. 2, pp. 35-41, 2009.

\section{REFERENCES}

Bershadskii, A. (2006). Isotherms clustering in cosmic microwave background. Physics Letters A, 360, 210216.

Bershadskii, A., and K.R. Sreenivasan (2003). Extended self-similarity of the small-scale cosmic microwave background anisotropy. Phys. Lett. A, 319, 21-23.

Bershadskii, A., and K.R. Sreenivasan (2002). Multiscaling of cosmic microwave background radiation. Phys. Lett. A, 299, 149-152.

Chernin, A.D., I.D. Karachentsev et al. (2007). Detection of dark energy near the Local Group with the Hubble Space Telescope. arXiv:astro-ph/0706.4068v1.

Gahm, G. et al. (2007). Globulettes as seeds of brown dwarfs and free-floating planetary-mass objects. $A J$, 133, 1795-1809.

Gibson, C.H. (2008). Cold dark matter cosmology conflicts with fluid mechanics and observations. J. Applied Fluid Mech., Vol. 1, No. 2, pp 1-8, 2008, arXiv:astro$\mathrm{ph} / 0606073$.

Gibson, C.H. (2006). The fluid mechanics of gravitational structure formation. astro-ph/0610628.

Gibson, C.H. (2005). The first turbulent combustion, Combust. Sci. and Tech., 177: 1049-1071, arXiv:astro$\mathrm{ph} / 0501416$.

Gibson, C.H. (2004). The first turbulence and the first fossil turbulence. Flow, Turbulence and Combustion, $72,161-179$.

Gibson, C.H. (2000). Turbulent mixing, diffusion and gravity in the formation of cosmological structures: The fluid mechanics of dark matter. J. Fluids Eng., $122,830-835$.

Gibson, C.H. (1996). Turbulence in the ocean, atmosphere, galaxy and universe. Appl. Mech. Rev., 49, no. 5, 299315 .

Gibson, C.H. and R.E. Schild (2007, a). Interpretation of the Helix Planetary Nebula using Hydro-GravitationalDynamics: Planets and Dark Energy. arXiv:astroph/0701474.

Gibson, C.H. and R.E. Schild (2007, b). Interpretation of the Stephan Quintet Galaxy Cluster using HydroGravitational-Dynamics: Viscosity and Fragmentation. arXiv[astro-ph]:0710.5449.

Gibson, C.H. and R.E. Schild (2002). Interpretation of the Tadpole VV29 Merging Galaxy System using HydroGravitational Theory. arXiv:astro-ph/0210583.

Hutsemekers, D. et al. (2005). Mapping extreme-scale alignments of quasar polarization vectors. $A \& A 441$, 915-930.

Jeans, J.H. (1902). The stability of spherical nebula. Phil. Trans., 199A, 0-49.
Krauss, L.M. and R.J. Scherrer (2008). The end of cosmology. Scientific American, 298 (3), 46-53.

Krauss, L.M. and R.J. Scherrer (2007). The return of a static universe and the end of cosmology, J. Gen. Rel. and Grav., 39, 10, 1545-1550, arXv:0704.0221.

Land, K. and J. Magueijo (2005). Phys. Rev. Lett. 95, 071301.

Longo, M.J. (2007). Evidence for a Preferred Handedness of Spiral Galaxies. $\operatorname{arXv}: 0707.3793$.

O'Dell, C.R., W.J. Henney and G.J. Ferland (2007). Determination of the physical conditions of the knots in the Helix nebula from optical and infrared observations. AJ, 133, 2343-2356, astro-ph/070163.

Pena, M. et al. (2004). A high resolution spectroscopic study of the extraordinary planetary nebula. $L M C$ N66, A\&A, 419, 583-592.

Perlmuter, S., G. Aldering et al. (1999). Measurements of $\Omega$ and $\Lambda$ from 42 high-redshift supernovae. ApJ, 517,565 .

Riess, A. G., A.V. Filippenko et al. (1998). Observational evidence from supernovae for an accelerating universe and a cosmological constant. AJ, 116, 1009.

Sandage, A. et al. (2006). The Hubble constant: a summary of the HST program for the luminosity calibration of Type Ia supernovae by means of Cepheids. ApJ, 653, 843.

Schild, R. (1996). Microlensing variability of the gravitationally lensed quasar. Q0957+561 A,B, ApJ, 464, 125.

Schild, R.E and C.H. Gibson (2008). Lessons from the Axis of Evil. axXiv[astro-ph]:0802.3229v2.

Toomre, A. and J. Toomre (1972). Galactic Bridges and Tails, ApJ, 178, 623.

Tran, H.D., M. Sirianni, M. and 32 others (2003). Advanced Camera for Surveys Observations of Young Star Clusters in the Interacting Galaxy UGC 10214. ApJ, 585, 750. 